\title{
Téoros
}

Revue de recherche en tourisme

\section{L'impact du libre-échange canado-américain sur le tourisme au Québec}

\section{Pierre Bellerose}

Volume 7, numéro 3, novembre 1988

Économie du tourisme

URI : https://id.erudit.org/iderudit/1080379ar

DOI : https://doi.org/10.7202/1080379ar

Aller au sommaire du numéro

Éditeur(s)

Université du Québec à Montréal

ISSN

0712-8657 (imprimé)

1923-2705 (numérique)

Découvrir la revue

Citer cet article

Bellerose, P. (1988). L’impact du libre-échange canado-américain sur le tourisme au Québec. Téoros, 7(3), 15-17. https://doi.org/10.7202/1080379ar d'utilisation que vous pouvez consulter en ligne.

https://apropos.erudit.org/fr/usagers/politique-dutilisation/ 


\section{L'impact du libre-échange canado-américain sur le

\section{1 - Les accords du libre-échange}

En 1947, le Canada et 22 autres pays signaient l'Accord général sur les tarifs douaniers et le commerce (GATT) qui les engageait dans un processus de libéralisation des échanges commerciaux. Les membres du GATT, aujourd "hui plus de 90 pays, en sont a leur huitième cycle de négociations commerciales multilatérales.

L'Accord général du GATT et son évaluation se fondent depuis l'origine sur quatre principes de base qui constituent les piliers de la réglementation commerciale internationale:

- commerce sans discrimination: tous les membres s'engagent à se traiter de la méme manière sans favoritisme ni exclusion arbitraire;

- seuls les droits de douane demeurent comme moyen de protection (élimination graduelle des quotas);

- stabilité pour le commerce;

- égalité entre tous et arbitrage par le GATT.

Le GATT a permis de réduire sensiblement les taux des tarifs douaniers depuis 40 ans. Ainsi, le taux moyen des tarifs douaniers sur les importations imposables aux États-Unis est passé de $24,1 \%$ pour la période 1940 1949 à $5,7 \%$ en $1978^{(2)}$.

Cependant, la grande exception au principe de non-discrimination du GATT est qu'il devient permis de signer des accords régionaux de coopération et d'intégration économique "à condition que les arrangements passés facilitent le commerce entre les pays intéressés sans opposer d'obstacles avec les. autres pays"."

$\mathrm{L}$ 'internationalisation du commerce et des investissements a donné lieu à la conclusion d'alliances commerciales touchant environ 80 pays développés ou en développement. C"est ainsi que l'intégration économique réalisée par l'entremise de la Communauté économique européenne (CEE) et l'Association européenne du libre-échange (AELE) a permis d'abolir de nombreuses barrières tarifaires (...) en plus d'ouvrir de vastes marchés (360 millions de consommateurs, ...). De nombreux pays en développement, en effet, sont membres d'associations régionales

\footnotetext{
- Consultant au centre d'tudes en tourisme et membre
} du Comite de redaction de Téoros comme la Communauté des Caraibes, la Communauté économique de l'Afrique de l'Ouest et l'Association pour l'intégration de l'Amérique latine, par exemple ${ }^{(3)}$.

\section{2 - L'Accord du libre-échange canado-américain}

\section{1 - Les objectifs}

Dès le début des négociations bilatérales avec les Etats-Unis, le Canada visait trois objectifs majeurs:

- un accès plus libre de ses exportations au marché des États-Unis. Il est à noter que les négociations entre le Canada et les États-Unis ont débuté au moment même où les deux Chambres du Congrès américain étaient en train d'étudier les Projets de loi les plus délibérément protectionnistes jamais proposés dans un grand pays industriel depuis les années ' 30 (bois d'oeuvre, etc.):

- un accès plus sûr aussi car les Canadiens voulaient être certains qu'après avoir investi sur le marché américain, ils ne seraient pas soumis aux caprices des organismes de réglementation américains ou aux interprétations des tribunaux;

- des dispositions spéciales pour les secteurs vulnérables ${ }^{(4)}$.

On remarque que les objectifs de l'Accord ne sont pas liés directement à la création d'emplois ou à une baisse des tarifs douaniers mais plutôt à la création d'un meilleur environnement pour les entreprises, ce qui sera propice à une plus grande croissance économique.

\section{2 - Les moyens}

$L$ 'Accord élimine presque toutes les barrières tarifaires et certaines non-larifaires (en ce qui concerne la promotion par exemple) entre le Canada et les Etats-Unis. Historiquement, les pays ont eu recours aux droits de douane et aux restrictions commerciales soit pour en tirer des recettes, soit pour stimuler la production locale ou encore pour protéger un secteur mou. Plusieurs études ont prouvé que ces obstacles au commerce peuvent, à court terme, se révéler efficaces, mais à long terme, ils deviennent inefficaces et ralentissent l'adaptation technologique et la croissance.

Un programme en trois étapes est prévu dans l'Accord du libre-échange pour l'élimination des droits de douane.
TABLEAU 1

Élimination des droits de douane

\begin{tabular}{ll}
\hline $\begin{array}{l}\text { Date prévue pour } \\
\text { l'elimination des } \\
\text { droits de douane }\end{array}$ & $\begin{array}{l}\text { Proportion des impor- } \\
\text { tations touchées par } \\
\text { l'elimination des droits } \\
\text { de douane }\end{array}$ \\
\hline ler janvier 1989 & $15 \%$ \\
ler janvier 1993 & $35 \%$ \\
ler janvier 1998 & $50 \%$ \\
\hline
\end{tabular}

Source: CEC, Le tremplin commercial. Une evaluation de l'Accord canadoaméricain,

Ottawa, 1988, p. 9.

\section{3 - Les conséquences}

Les impacts du libre-échange sont évalués très différemment selon la vision qu 'on a de l'économie.

Les macro-économistes (économistes, industriels, etc.) regardent et examinent les impacts selon une vue d'ensemble. Ils croient que le libre-échange clarifie les règles du jeu, et par conséquent, améliore le climat économique. De plus, ils estiment que certaines industries moribondes disparaîtront, ce qui aura pour effet d'éliminer le bois mort et les canards boiteux et de conserver uniquement les entreprises plus concurrentielles.

Tout ce processus devrait rendre l'économie canadienne et québécoise plus compétitives au niveau mondial et permettre vraisemblablement la création de quelque 250000 emplois (au Canada).

De plus, cette grande concurrence aura comme effet de réduire les prix et ainsi d'augmenter le revenu net des consommateurs.

Cette thèse est soutenue par le Conseil économique du Canada et par une foule d'économistes de diverses écoles (Bernard Landry, Pierre-Paul Proulx et Rodrigue Tremblay sans oublier Robert Bourassa et Jacques Parizeau) ${ }^{(5)}$

A ce niveau, toutes les études semblent montrer que le Canada sort gagnant. L'analyse du Conseil économique du Canada prévoit que le libre-échange créera 250000 emplois d'ici dix ans. Malgré les apparences, il faut dire que ces chiffres sont modestes; en dix ans de 1976 à 1986. il s'est créé 2,6 millions $d^{\prime}$ 'emplois d'un océan à l'autre ${ }^{(6)}$. 
Pour leur part, les micro-economistes (même si plusieurs des intéressés ne se reconnaissent pas sous ce vocable), tout en ne remettant pas en cause les principes généraux des macro-économistes, pensent que l'on surévalue les effets positifs du libre-échange et qu'on passe sous silence, un peu trop vite, ceriains aspects négatifs.

Les gains dans les industries gagnantes dépassent les pertes d'emplois dans des secteurs qui auront du mal à résister, comme le vêtement. Malgré les perspectives géneralement positives, on peut se demander ce qu'il arrivera aux travailleurs affectés.

Selon les micro-économistes (où l'on retrouve les interlocuteurs des centrales syndicales regroupées dans la Coalition québécoise d'opposition au libre-échange), ce phénomène se répercutera à l'intérieur d'une même industrie. En effet, si bien des entreprises profiteront de l'entente pour faire un bond en avant, d'autres auront du mal à s'ajuster à la concurrence. Encore là, il y aura des bouleversements et, même à l'intérieur de chaque entreprise, les exigences $d v$ libre-echange pousseront a la modernisation. a la rationalisation et à la spécialisation ${ }^{(7)}$. L'entente nécessitera donc d'importants investissements dans les politiques de maind'oeuvre qui se répercuteront dans chacun des secteurs, le champ récréotouristiqué ne faisant pas exception à la règle.

Nous nous retrouvons donc devant deux types d'analyse où l'on fait référence à des dimensions différentes d'un même problème. Cependant, les macro-économistes ont l'avantage de s'appuyer sur plusieurs études alors que les micro-economistes doivent $s$ 'en tenir à des scénarios.

\section{3 - La place du tourisme dans le libre-échange}

Comme le soulignait récemment Louis Jolin, l'industrie touristique, tant américaine que canadienne évolue déjà dans un contexte de libre-bchange et, à ce titre, bénéficie de plusieurs années des opportunités qu'offre la libéralisation des echanges ${ }^{(8)}$.

Pour la premieres fois, un Accord du libreéchange accorde une place non négligeable au commerce des services. A la fin de cette section de l'Accord, on retrouve une annexe sectorielle (annexe 1404.B) sur le tourisme.

L'Accord stipule qu 'une province ou un état doit accorder aux personnes de l'autre pays un traitement non moins favorable que celui accordé à ses résidents (principe du traitement national). L'Accord reconnaît également le droit de présence commerciale et le droit d'établissement aux foumisseurs de services de l'autre pays.

$L^{\top}$ Accord $n$ 'impose aucune obligation visant les subventions et les pratiques des gouvernements (article 1402.9 de l'Accord) dans le domaine des services. Ainsi, il semble qu'il $n$ 'y ait aucune restriction pour les gouvernements (de tous niveaux) en ce qui concerne l'intervention financière.

\section{1 - L'annexe Tourisme}

L'annexe 1404-B de I'Accord du librechange porte exclusivement sur le tourisme. L'énumération exhaustive des services de tourisme qui sont visés vient définir de façon précise la portée de l'Accord. Ainsi, la catégorie des services de tourisme comprend les activités liées au tourisme, dans les cas suivants: les services d'agences de voyages et les services connexes, les firmes-conseils, la délivrance d'une assurance-voyage, tous les modes de transport international de passagers, le logement y compris les hôtels, motels et maisons de rapport, les services de guides/interprètes, la location de voitures, les services d'organisation et d'appui à l'occasion de congrès internationaux, les services liés aux terrains de camping et aux parcs à roulottes, les services liés aux parcs d'amusement, les attractions touristiques commerciales et les services touristiques de nature financière.

Pour les entreprises qui fournissent ces services, les gouvernements se sont entendus pour que les compagnies américaines qui viennent s'établir au Canada bénéficient des mémes conditions que les entreprises locales et vice versa (traitement local).

De plus, les deux gouvernements reconnaissent que chaque pays peut faire officiellement de la promotion sur le territoire de l'autre pays, ce qui comprend la réalisation de promotions communes avec des entreprises de tourisme de chaque partie ainsi qu'avec les gouvernements des provinces, des états et des administrations locales.

Aussi, l'Accord stipule que l'imposition et/ou la pereeption de droits, soit de frais de départ, soit de frais d'arrivée des touristes entravent la libre circulation des personnes. Mais si jamais ils sont imposés, ils devront être compatibles avec le principe du traitement national et leur montant sera limité au coût approximatif des services rendus.

On doit souligner qu une annexe spécifique au transport a été exclue à la toute dernière minute à cause d'un différend portant entre autres sur le transport maritime. L'Accord ne s'applique pas au secteur des transports maritimes, aérien et ferroviaire ainsi que par camions et par autobus. On s'en remet aux arrangements existants: à titre d'exemples, signalons les divers accords bilatéraux sur le transport aérien et ceux négociés dans le cadre de 1'O.A.C.I. Cette exclusion est de taille et limite la portée de l'Accord dans le secteur du tourisme.

\section{2 - Les voyages d'affaires}

Les deux gouvernements ont adopté des règlements relatifs à l'immigration pour faciliter les voyages d'affaires. Le chapitre 15 de l'Accord régit les séjours de diverses catégories de gens d'affaires.

L'Accord prévoit l'accès réciproque aux gens d'affaires du Canada et des ÉtatsUnis au marché de l'autre pays. Les lois et les règlements nationaux applicables seront libéralisés et les formal ités d'entrée seront plus simples et rapides. Parmi les gens d'affaires qui bénéficient d'une telle libéralisation (ex.: sans avoir à obtenir un permis de travail pour être admis dans l'autre pays), on retrouve le personnel du secteur du tourisme (agents de voyages, organisateurs de voyages ou guides touristiques) qui assiste ou participe à des congrès ou qui est chargé d'un circuit qui a commencé dans son propre pays. ${ }^{(9)}$

Les procédures allégées devraient favoriser les voyages d'affaires d'autant plus que $I^{\top}$ Accord du libre-échagne amènera un accroissement des echanges commerciaux entre les deux pays, donc une augmentation des voyages d'affaires et des congrès et, par conséquent, des effets bénéfiques pour les fournisseurs de services à ces catégories de voyageurs, tels que les hôtels, les restaurants et les transporteurs aériens.

\section{3 - Les impacts indirects}

En plus de l'annexe spécifique portant sur le tourisme, diverses autres mesures peuvent ềtre prises en considération et être analysées en fonction de leurs impacts possibles dans le champ récréotouristique

Comme l'écrivait Louis Jolin, la suppression progressive des barrieres tarifaires, des droits de douane sur les produits peut avoir un impact certain sur le coüt du produit touristique: certains produits almentaires, des boissons alcoolisées reviendront moins chers et pourront contribuer à la baisse des prix au consommateur.

Comme autre mesure, la libéralisation des investissements pourra certes avoir des incidences sur la propriété des infrastructures touristiques (notamment les grandes chaines hôtelières).

À l'avenir, les investisseurs canadiens aux États-Unis et les investisseurs américains au Canada seront assujettis aux mêmes règlements que les investisseurs nationaux en matiere de constitution d'une nouvelle entreprise.

Le Canada conserve encore cependant le droit d'examiner les acquisitions effectuées au Canada mais il accepte de relever graduellement le seuil d'examen des acquisitions directes. Le seuil d'examen des acquisitions directes sera porté en quatre étapes de $5 \mathrm{mil}-$ lions de dollars qu'il est actuellement à 150 millions de dollars d'ici 1992. Il existe donc un risque d'implantarion d'imporiantes infrastructures américaines au detriment de celles québécoises dejà existantes.

De plus, mème si les industries culturelles sont exclues de l'Accord, les parties se gatdent un droit de réplique contre toute politique culturelle qu'elles jugeraient protectionniste. Cependant, les industries culturelles québécoises sont fortement subventionnées compte tenu de la petitesse de notre marché. Pour ce secteur, si les subventions étaient réduites au nom d'une plus grande équité commerciale, cela pourrait avoir un aspect catastrophique. 


\section{4 - L'impact de I'Accord canado-américaín sur le tourisme}

L'industrie récréotouristique en est une difficile à analyser, compte tenu de sa complexité. Mais, en plus, vouloir établir une certaine corrélation avec un phénomène aussi tentaculaire que le libre-échange tient du périlleux.

Cependant, en s'en tenant à des concepts simples et en effectuant des regroupements à l'intérieur des études économiques disponibles, il est possible de tirer certaines conclusions intéressantes.

Ainsi, au niveau des avantages, l'Accord du libre-échange a le mérite de créer un meilleur environnement pour les entreprises puisqu'il clarifie les régles du jeu en éliminant l'ensemble des barrières tarifaires.

De plus, l'Accord amènera une meilleure performance économique qui se traduira par une augmentation du revenu net du consommateurs. Une des hypothèses des économistes weut que ce gain (du revenu net des consommateurs) ira, dans une large mesure, à l'industrie récréotouristique. Ainsi, le tableau 2 montre les impacts du libre-échange sur l'emploi au Canada et au Québec pour les principales industries. Les résultats indiquent un gain net de 251000 emplois (97000 au Québec) sur dix ans.

En ce qui concerne le secteur récréotouristique, les quatre industries concemés (holellerie et loisirs, restauration et récréation. materiel de transport et produirs manufacfuriers divers) comptabilisent un gain net (en 10 ans) de 27900 emplois pour le Canada et de 9600 pour le Québec $34,4 \%$ du total canadien). Ce nombre représente $11,1 \%$ du total pour le Canada (27 900/251 000) et $9,9 \%$ du total pour le Québec (9600/ 97300 ).

Bien sûr, le libre echange aura des impacts négatifs mais ils semblent moins grands que les impacts positifs. Ainsi, certains secteurs à l'abri de la concurrence depuis longtemps, grăce à des tarifs douaniers protectionnistes (ce qui touche peu au secteur récréotouristique), auront à effectuer une rationalisation qui amènera sans doute une réduction du nombre d'emplois.

Signalons que l'industrie récréotouristique devra probablement faire face à une certaine baisse d'activités, au moins durant les premières années d'application de l'Accord, étant donné qu'un nombre accru de Canadiens voyageront aux États-Unis pour profiter de la possibilité d'importer des marchandises en franchise.

Il demeure quelques points d'interrogation cependant qui risquent d'hypothéquer la nature du produit touristique québécois. Par exemple, toute la gamme des industries culturelles qui, en principe, est exclue de l'Accord, pourrait être affectée au nom d"une plus grande équité commerciale.

TABLEAU 2

Projection des flux de l'emploi au Canada et au Quebec découlant de I'entente sur le libre-cchange, selon l'industrie, 1998

\begin{tabular}{|c|c|c|c|c|}
\hline & $\begin{array}{l}\text { CANADA } \\
\text { Changement } \\
\text { net }\end{array}$ & $\begin{array}{l}\text { o de } \\
\text { changement }\end{array}$ & $\begin{array}{l}\text { QUÉBEC } \\
\text { Changement } \\
\text { net }\end{array}$ & $\begin{array}{l}\text { \% du total } \\
\text { canadien }\end{array}$ \\
\hline \multicolumn{5}{|l|}{ Impact positif } \\
\hline $\begin{array}{l}\text { Commerce de détail } \\
\text { Construction } \\
\text { Services financiers et immobiliers } \\
\text { Hotellerie et restauration } \\
\text { Service aux entreprises } \\
\text { Commerce de gros } \\
\text { Services personnels et divers } \\
\text { Transport et entreposage } \\
\text { Imprimerie et edition } \\
\text { Agriculture } \\
\text { Aliments et boissons } \\
\text { Education et sante } \\
\text { Mines } \\
\text { Metaux primaires } \\
\text { Loisirs et récréation } \\
\text { Matériel de transport } \\
\text { Autres }\end{array}$ & $\begin{array}{r}59000 \\
37500 \\
30300 \\
23600 \\
20000 \\
15720 \\
10300 \\
8800 \\
8400 \\
7200 \\
6400 \\
5900 \\
5000 \\
5000 \\
4600 \\
600 \\
12500 \\
\end{array}$ & $\begin{array}{l}+3.1 \\
+5.07 \\
+3.02 \\
+2.12 \\
+1.17 \\
+2.34 \\
+0.67 \\
+1.77 \\
+6.08 \\
+1.80 \\
+2.00 \\
+3.46 \\
+1.69 \\
+2.93 \\
+1.71 \\
+0.30\end{array}$ & $\begin{array}{r}14700 \\
8700 \\
7400 \\
5200 \\
1600 \\
3600 \\
2600 \\
1700 \\
2100 \\
1200 \\
1500 \\
1200 \\
200 \\
1300 \\
4500 \\
100 \\
5200 \\
\end{array}$ & $\begin{array}{l}25 \% \\
23.2 \% \\
24.4 \% \\
22.0 \% \\
8 \% \\
23 \% \\
25.3 \% \\
19.3 \% \\
25 \% \\
16.6 \% \\
23.4 \% \\
20.4 \% \\
4 \% \\
26 \% \\
98 \% \\
16.6 \%\end{array}$ \\
\hline \multicolumn{5}{|l|}{ Impact négatif } \\
\hline $\begin{array}{l}\text { Produits électriques } \\
\text { Caoutchouc et matières plastiques } \\
\text { Textiles } \\
\text { Produits manufacturiers divers } \\
\text { Autres }\end{array}$ & $\begin{array}{l}-4500 \\
-1642 \\
-1266 \\
-900 \\
-1441\end{array}$ & $\begin{array}{l}-3.38 \\
-1.72 \\
-2.05 \\
-1.40\end{array}$ & $\begin{array}{l}-1200 \\
-\quad 400 \\
=\quad 600 \\
=\quad 200 \\
=\quad 600\end{array}$ & $\begin{array}{l}26.6 \% \\
24.4 \% \\
47.4 \% \\
22.2 \%\end{array}$ \\
\hline TOTAL: & +251000 & +1.8 & +97300 & $+38.8 \%$ \\
\hline
\end{tabular}

Source: BELLEROSE. Pierre avec la collaboration de PELLETIER. Jean, Le libre-6change et le champ récrbotouristique: un secteur gagnant, Montréal: 1988. Les dossiers du CET, p. 33.

Globalement, le champ récréotouristique sortira gagnant du libre-échange à cause principalement de trois phénomènes:

1- augmentation du revenu des consommateurs;

2- réduction du coût du produit récréotouristique;

3- augmentation du tourisme d'affaires.

\section{Conclusion}

L'Accord de libre-echange devient uñ autre élément à prendre en compte par les analystes et surtout par les intervenants (tant privés que publics) en termes de planification et de marketing. Est-ce que ces derniers sauront évaluer ce potentiel (puisque, pour l'instant, on ne peut parler d'autres choses)? Estce qu'ils pourront amener les consommateurs à utiliser leur revenu discrétionnaire pour des produits récréotouristiques québécois?

Jusqu'ici, les indices disponibles nous laissent penser que l'industrie récréotouristique québécoise sortira grandie du libre-echange uniquement si elle réussit à canaliser ce nouvel argent disponible pour elle méme et ainsi se démarquer de la concurrence. Pour ce faire, les intervenants devront assurer un leadership renouvelé pour faire face aux défis des années '90. Mais, plus encore, le monde touristique québécois devra aussi composer avec d'autres facteurs importants qui viendront modifier en profondeur les règles du jeu (vieillissement de la population, augmen- tation de la proportion des groupes ethniques, etc.).

En fait, toutes ces dimensions auront des impacts considérables sur les habitudes de loisir et de tourisme. Ces répercussions seront beaucoup plus importantes que le libre-échange qui a des effets somme toute relativement minimes. Il importe de bien positionner ces priorités et de ne pas passer à côté de l'essentiel. A trop wouloir observer l'arbre, on en oublie la forêt. $f$

\section{Notes explicatives}

(1) Source: Cet article est largament inspired de ta recher. che La libre-tehange ot le champ récretouristi. que: un secteur gagnant. de Pierre Bellerose anoc la collaboration de Jean Pelletier.

121 LANDAY, Bernard, Commerce sans trontibres. Le sens du libre echange, Montreal: Quebec/Ametique, 1987. DP. 120-121.

[3) CONSEIL ECONOMIQUE DU CANADA, Le tremplin commercial. Une evaluation de l'accord canado americain, Ottawa: CEC, 1908, p. 3.

(4) Ces informations ont att puistes aux papes 5 et 6 du document du CEC: Le tremplin commercial.

151 A cet effet, voir l'article de Marie Tilson, 250 economistes appuient le libre fchange. Le Devoir, 26 auril 1998 .

(6) DUBUC, Alain, Le libretehange: une aftaine de jobs. Avenir, fewripr-mars 1988.

17) Selon Alain Dubuc et, avec plus de vigueur, ta Coalition quebécoise d'opposition au libre-echange.

[8] JOLIN, Louis, L'Accord du libre-échange Canada/tetats-Unis; ses consequences sur be towrisme, janvier 1988, p. 10.

[9l Idem, p. 14 . 